\section{Hemorrhagic acute pancreatitis following percutaneous transhepatic biliary drainage: a rare and serious complication}

\section{Introduction}

PTBD is an invasive procedure and hence associated with complications, some of which may be fatal. The most common complications include bleeding, cholangitis and sepsis; however several studies have reported a complication rate comparable to endoscopic retrograde cholangiopancreatography (ERCP). Pancreatitis is a rare but underreported complication of PTBD. However, asymptomatic hyperamylasemia is not uncommon following PTBD. Hemorrhagic pancreatitis following PTBD is a rather rare and serious complication.

\section{Case report}

\section{Case 1}

A sixty-year-old male, diagnosed case of hilar cholangiocarcinoma was referred to our intervention suite for transhepatic decompression of the biliary system. The patient had increasing jaundice, fever with chills, itching and severe anorexia. Urgent PTBD was required to relieve cholangitis.
Investigations revealed haemoglobin of $12 \mathrm{~g} \%$, platelet count of 120,000/ $\mu 1$, prothrombin time of 15 seconds. Preprocedure antibiotics were administered. Since she had hilar obstruction, both right and left systems were drained by internal-external drainage without any intraprocedural complications (Figure 1). Post procedure, the patient was kept under observation; and antibiotic coverage was continued. The next day the patient complained of severe epigastric pain with radiation to back with associated nausea and vomiting. She also had abdominal distension and ultrasound confirmed the presence of ascites. Diagnostic tap of ascitic fluid revealed presence of hemorrhagic tap. Serum amylase level was 3,000 IU/ml. Based on clinical findings, high serum amylase level, and hemorrhagic ascitic tap, a diagnosis of hemorrhagic pancreatitis was made. The patient was started on conservative management for pancreatitis. He was kept nil per oral, given parenteral fluids, analgesics and antibiotics. The patient improved and showed signs of recovery with stable vital signs and no systemic complications.

\section{Case 2}

A 50-year-old female presented with a one-year history of progressively increasing jaundice, weight loss and pruritis. She had a history of gallstones, diagnosed six years back. She had occasional episodes of vomiting. On examination, the patient was poorly built. There was pallor and deep yellowish discoloration of the skin. On abdominal examination, no lump

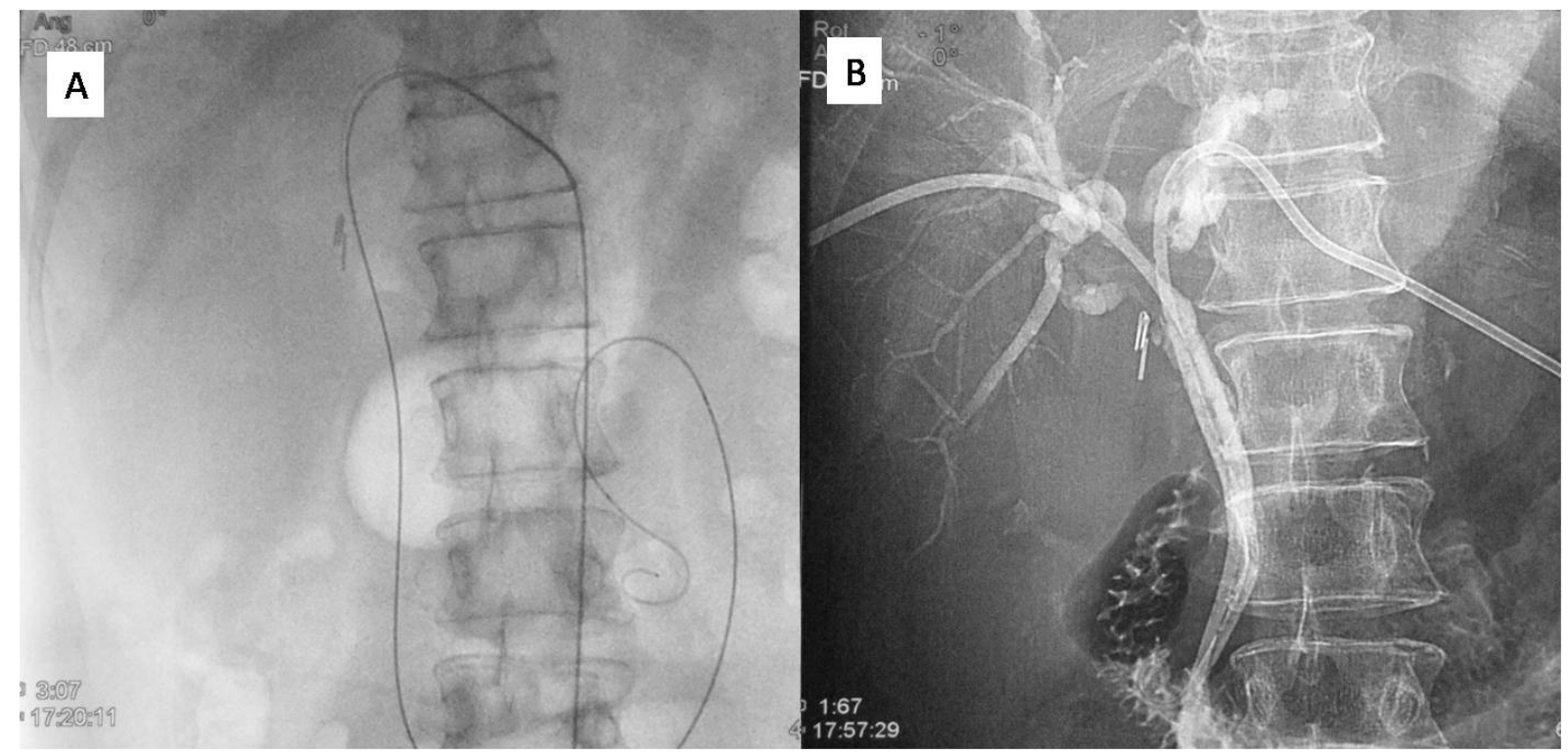

Figure 1: Fluoroscopic spot images showing, metallic guidewire placed from the left side biliary system across the ampula of vater into the duodenum (A), two ring biliary catheters $(8 \mathrm{~F})$ one each from right and left side biliary system placed across the ampulla of vater into the duodenum acting as internal-external drainage (B). 
was palpable. The gallbladder was non-palpable. There was no tenderness in the right hypochondrium. Biochemical profile revealed elevated total bilirubin which was $12 \mathrm{mg} \%$ with direct bilirubin $9 \mathrm{mg} \%$. Hemoglobin was $9 \mathrm{~g} \%$, platelet count of $180,000 / \mu 1$ and normal total and differential leukocyte counts. Abdominal ultrasound revealed bilateral intrahepatic biliary radical dilatation with block at the primary confluence. No definite mass could be seen. Bilateral secondary confluences were patent. There was cholelithiasis; however the common bile duct (CBD) was of normal calibre. A provisional diagnosis of hilar cholangiocarcinoma was considered and patient was sent for PTBD procedure. A left-sided approach was used and an $8 \mathrm{~F}$ ring biliary catheter was put across the ampulla of Vater and patient left to external drainage (Figure 2). The following day, the patient complaint of severe epigastric pain with several episodes of vomiting. Serum amylase was elevated. A diagnosis of acute pancreatitis complicating PTBD was made and the patient was managed conservatively. Also, ring biliary catheter was replaced by simple pigtail catheter and the tip of the catheter is placed proximal to the ampulla of vater.

Magnetic resonance cholangiopancreatography (MRCP) was performed to evaluate the level of obstruction, ductal anatomy and the severity of pancreatitis. MRCP showed a block at primary confluence with no involvement of secondary confluences (Figure 3A). No mass was noted. No variant ductal anatomy was found. Gallbladder was filled with several calculi. Both the CBD and main pancreatic duct were prominent with no filling defects. Pancreas was diffusely bulky with minimal peripancreatic fluid (Figure 3B). Thus a diagnosis of hilar cholangiocarcinoma with cholelithiasis and mild acute pancreatitis secondary to PTBD was established.

\section{Discussion}

The percutaneous transhepatic route is an important approach to the management of biliary obstruction. It is preferred over the endoscopic retrograde route in patients with hilar biliary obstruction. It may also be required in unresectable disease and where there is failure of endoscopic approach because of anatomical variations, inflammatory processes, in patients who have undergone Billroth II gastrectomy, biliojejunostomy after gastrectomy or resection of pancreatic head (Whipple procedure). The reported prevalence of major complications following PTBD is 4-25\%..$^{1-3}$ Various complications associated with PTBD include bleeding, acute cholangitis, sepsis, biloma, intrahepatic haematoma, biliovenous fistula, biliopleural fistula, pneumothorax, perforation and peritonitis. ${ }^{1}$ A rare complication is acute pancreatitis. Endoscopic retrograde cholangiopacreatography carries a well-recognized risk of acute pancreatitis. ${ }^{4}$ Similarly, antegrade manipulation of the biliary

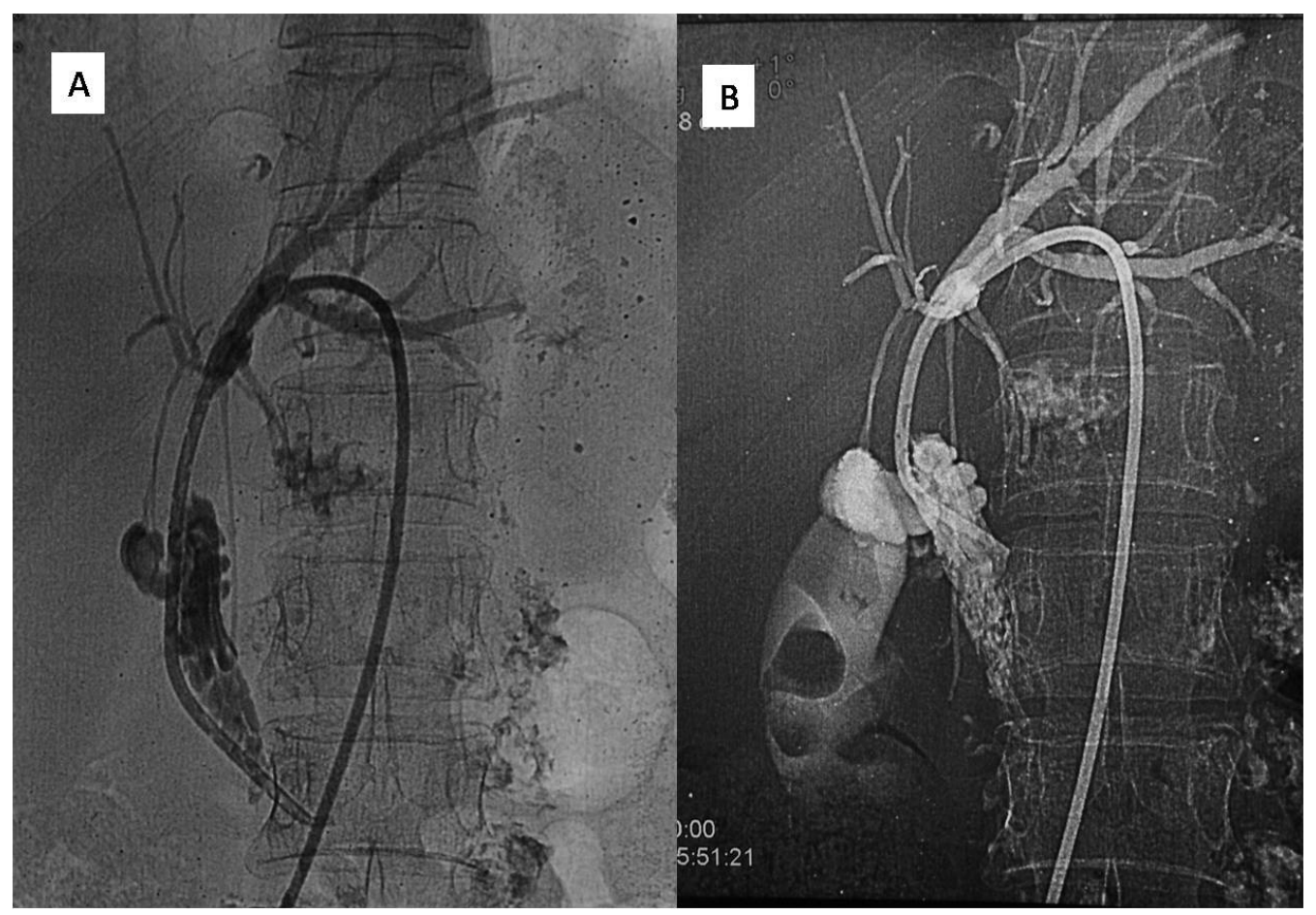

Figure 2: Fluoroscopic Spots images showing, a 8 F ring biliary catheter is placed across the hilar stricture and the ampulla of vater into the duodenum (A). Following the development of haemorrhagic pancreatitis, the ring biliary catheter was replaced with simple pigtail catheter and tip of catheter is placed proximal to Ampulla of vater (B). Note: Multiple filling defects are seen in gall bladder in the cholangiographic spot image. 


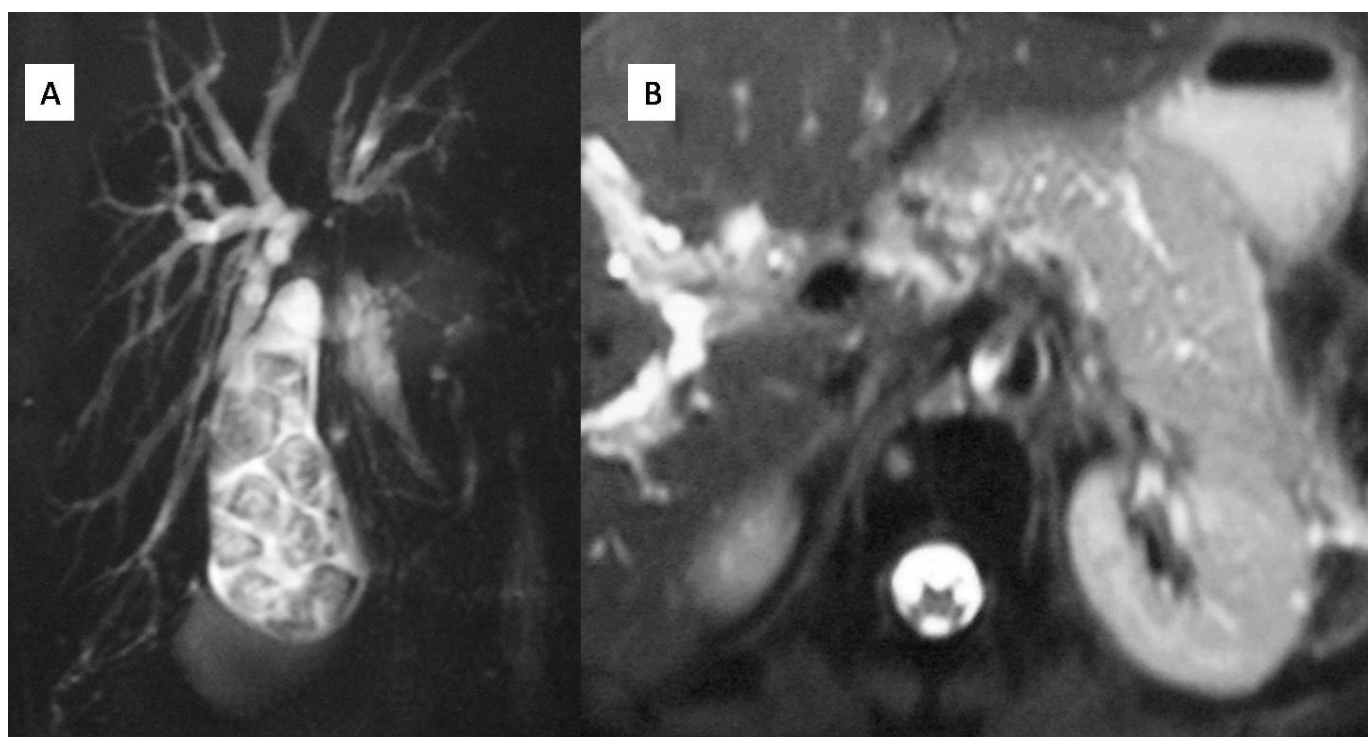

Figure 3: Thick slab coronal MRCP image showing, the dilatation of bilateral intrahepatic biliary radicles with separation of right and left ducts at the primary confluence with multiple filling defects in gall bladder (A). Axial T2 weighted FSE (TR 4000, TE 90) MR image at the level of pancreas reveals diffusely enlarged pancreas with prominent MPD and minimal peripancreatic fluid suggestive of mild acute pancreatitis (B).

tree is associated with an increased risk of acute pancreatitis. ${ }^{5}$

SHALIMAR $^{3}$

A review of literature by Al-Bahrani AZ et al found 11 reported cases of pancreatitis as a complication of percutaneous transhepatic interventions (PTBI) and documented five patients with pancreatitis following PTBI in their original research. ${ }^{6} \mathrm{An}$ interesting observation was a risk of pancreatitis comparable to ERCP. This risk is greater with distal interventions than with proximal procedures. Similarly in our patients, PTBD catheters were placed across the ampulla of Vater into the duodenum. The reason why distal biliary manipulations increase the risk of pancreatitis is similar to gallstone pancreatitis with contribution from both mechanical obstruction to ampullary drainage and a component of inflammatory edema. Furthermore, placement of stent increases the likelihood of developing pancreatitis. $^{7}$ Most cases of pancreatitis are diagnosed based on elevation of serum amylase level. Those who develop clinical symptoms have mild pancreatitis. Very few patients develop severe pancreatitis, as was experienced by case 1 in our report. So it is important to keep this complication of PTBD in patients who undergo PTBD for unresectable malignant biliary obstruction in whom tubes are placed across the ampulla of vater.

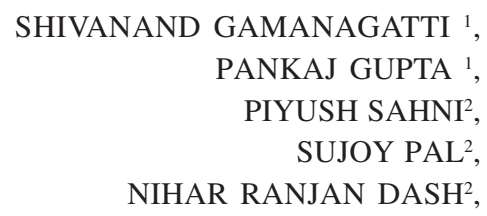

Correspondence: Dr Shivanand Gamanagatti
Department of Radiodiagnosis ${ }^{1}$,

Department of Gastrointestinal Surgery ${ }^{2}$ and Department of Gastroenterology, All India Institute of Medical Sciences,

New Delhi - 110029, India Email:shiv223@gmail.com

\section{References}

1. Mueller PR, van Sonnenberg E, Ferrucci JT. Percutaneous biliary drainage: technical and catheter related problems in 200 procedures. Am J Radiol. 1982;138:17-23.

2. Yee AC, Ho CS. Complications of percutaneous biliary drainage: benign vs. malignant diseases. Am J Radiol. 1987;148:1207-9.

3. Funaki B, Zaleski GX, Straus CA, Leef JA, Funaki AN, Lorenz $\mathrm{J}$, et al. Percutaneous biliary drainage in patients with nondilated intrahepatic bile ducts. Am J Radiol. 1999;173:1541-4.

4. Cohen SA, Siegel JH, Kasmin FE. Complications of diagnostic and therapeutic ERCP. Abdom Imaging. 1996;21:385-94.

5. Paganini AM, Feliciotti F, Guerrieri M, Tamburini A, De Sanctis A, Campagnacci R, et al. Laparoscopic common bile duct exploration. $J$ Laparoendosc Adv Surg Tech A. 2001;11:391-400.

6. Al-Bahrani AZ, Holt A, Hamade AM, Abid GH, Laasch HU, O'Shea SJ, et al. Acute pancreatitis: an under-recognized risk of percutaneous transhepatic distal biliary intervention. $H P B$ (Oxford). 2006;8:446-50.

7. Van-Steenbergen W, Van-Aken L, Ponette E. Acute pancreatitis complicating the insertion of a self-expandable biliary metal stent. Endoscopy. 1992;24:440-2. 